\title{
ANÁLISE DO CONHECIMENTO SOBRE DSTs/AIDS ENTRE ADOLESCENTES EM GOIÂNIA, GOIÁS
}

\author{
Marielton dos Passos CUNHA ${ }^{1}$ ** \\ Bruno Francesco Rodrigues de OLIVEIRA ${ }^{2} \neq$ \\ Izadora Cristina Moreira de OLIVEIRA ${ }^{3}$ \\ Layanny Kelly Silveira PRAXEDES ${ }^{4}$ \\ Ângela Adamski da Silva REIS ${ }^{5}$
}

\begin{abstract}
${ }^{1}$ Biomédico. Doutorando em Bioinformática pela Universidade de São Paulo (USP). marieltondospassos@gmail.com
${ }^{2}$ Biomédico. Professor Assistente do Departamento de Bioquímica e Biologia Molecular (DBBM) do Instituto de Ciências Biológicas (ICB) da Universidade Federal de Goiás (UFG). bfro.francesco@ gmail.com

${ }^{3}$ Biomédica. Mestranda em Genética e Melhoramento de Plantas pela UFG. izadorabiomed@gmail.com

${ }^{4}$ Biomédica. Mestranda em Biologia da Relação Parasito-Hospedeiro pela UFG. layanny_kelly@ hotmail.com

${ }^{5}$ Biomédica. Professora Adjunta do DBBM do ICB da UFG. angeladamski@gmail.com
\end{abstract}

\# Estes autores contribuíram igualmente para este trabalho.

* Autor Correspondente. e-mail: marieltondospassos@gmail.com

Recebido em: 24/03/2016 - Aprovado em: 29/08/2016 - Disponibilizado em: 18/12/2016

\section{RESUMO}

A adolescência é um período de mudanças, com transformações responsáveis pelo início da vida social e sexual. A sexualidade constitui uma das dimensões do ser humano em diversos aspectos, sobretudo o reprodutivo e emocional. Neste sentido, o presente estudo objetivou analisar conhecimentos, atitudes e práticas sexuais associadas às DSTs / AIDS entre adolescentes de Goiânia, Goiás. De 241 indivíduos, 70,9\% eram mulheres. A maioria dos participantes da pesquisa demonstraram possuir conhecimento sobre as vias de transmissão sexual, parenteral e vertical e, em geral, os adolescentes apresentaram ter um bom conhecimento sobre métodos de prevenção frente às DSTs. No entanto, um conhecimento inadequado sobre a biologia do agente etiológico da AIDS foi observado em $8,7 \%$ da população de estudo. Os resultados ressaltam a importância de campanhas de educação conscientização sobre as DSTs, principalmente no ambiente escolar, que têm se apresentado como eficazes entre os jovens, que estão cada vez mais informados sobre o controle e prevenção de DSTs.

Palavras-chave: Doenças Sexualmente Transmissíveis. AIDS. Adolescentes. Conscientização. Prevenção.

\section{ANALYSIS OF KNOWLEDGE ABOUT DSTS/AIDS AMONG TEENAGERS IN GOIÂNIA, GOIÁS}

\begin{abstract}
Adolescence is a period of changes and transformations responsible for the beginning of social and sexual life. Sexuality is one of the dimensions of the human being that involves various aspects, especially reproductive and emotional ones. In this sense, the present study aimed to analyze
\end{abstract}


knowledge, attitudes and sexual practices associated with DSTs / AIDS among adolescents in Goiânia, Goiás. From 241 participants, $70.9 \%$ were women. The majority of the participants from the research were found to have knowledge about sexual, parenteral and vertical transmission routes and, in general, teenagers have presented to have a good knowledge about STDs preventing methods. However, an inadequate knowledge about the etiologic agent biology of AIDS was observed in $8.7 \%$ from the study population. The results highlight the importance of education and awareness campaigns about STDs, especially at school, which have been presented as effective among young people, who are increasingly informed about the control and prevention of STDs.

Keywords: Sexually Transmitted Diseases. AIDS.Teenagers. Prevention. Awareness.

\section{INTRODUÇÃO}

As doenças sexualmente transmissíveis (DSTs) constituem um grupo de doenças que se disseminam principalmente pelo contato íntimo, englobando uma série de infecções causadas por vírus, fungos, protozoários e bactérias, sendo a via preferencial de transmissão a via sexual (BARBOSA et al., 1990; BRÊTAS, 2009). Apesar da população de adolescentes e jovens adultos constituírem apenas $25 \%$ da população sexual ativa, é a que representa aproximadamente $50 \%$ dos novos casos de DSTs adquiridas (SIRACUSANO et al., 2014).

A síndrome da imunodeficiência adquirida (SIDA), ou do inglês Acquired Immunodeficiency Syndrome (AIDS) foi descrita em meados de 1970, após casos relatados nos Estados Unidos, Haiti e África Central (SHARP et al., 1999) e atualmente constitui uma das principais DSTs. Desde o início do avanço dessa doença, estratégias que visam a diminuição de sua disseminação têm sido amplamente divulgadas. Uma medida, que vai ao encontro dessa necessidade, trata- se da inserção da Educação Sexual no âmbito escolar, sendo definida como o "processo de aquisição de informação e formação de atitudes e crenças sobre sexo, identidade sexual, relacionamentos e intimidade" (BERGER et al., 2008).

A adolescência é um período de mudanças biopsicossociais, tais como maturação dos caracteres sexuais secundários, independência socioeconômica e emocional dos pais, elaboração da identidade pessoal e sexual, aquisição do pensamento abstrato, exercício da sexualidade, intimidade e afetividade (CARLINI-COTRIM; GAZALCARVALHO; GOUVEIA, 2000; ROMERO et al., 2007). As transformações culturais ocorridas ao longo dos anos em nossa sociedade têm contribuído para a assimilação de novos valores e atitudes frente às questões de sexualidade, influenciando diretamente o comportamento dos adolescentes. A sexualidade é, portanto, elemento significante na formação da identidade do adolescente, manifestada por múltiplas identificações, como da imagem corporal, da descoberta do outro como objeto de amor ou desejo e a 
descoberta de si e das relações com os familiares, grupos e profissionais (VIEIRA et al., 2006).

$\mathrm{Na}$ atualidade, observa-se que a atividade sexual inicia-se cada vez de forma mais precoce, e pesquisadores e profissionais da área de Saúde Pública vem dispensando uma atenção especial à população jovem, pois a mesma apresenta-se bastante vulnerável aos riscos e problemas relativos à saúde do comportamento sexual, em diversos aspectos (GERHARDT; NADER; PEREIRA， 2008; KINCAID et al., 2012). Assim, o objetivo deste trabalho foi avaliar o conhecimento de adolescentes sobre sexualidade, métodos contraceptivos e doenças sexualmente transmissíveis entre estudantes das redes pública e privada de ensino da cidade de Goiânia, Goiás.

\section{MATERIAIS E MÉTODOS}

Inicialmente, na primeira etapa, foram distribuídas 500 cartilhas informativas sobre as DSTs e métodos contraceptivos durante um evento destinado a adolescentes (Espaço das Profissões da UFG - 2013) na Universidade Federal de Goiás (UFG). A cartilha foi elaborada por estudantes membros da Liga Acadêmica em Diagnóstico Molecular (LADMOL) do curso de Biomedicina do Instituto de Ciências Biológicas (ICB) da UFG.

Entre as 500 cartilhas distribuídas, adolescentes responderam um questionário

semi-estruturado, autopreenchível e anônimo. O questionário foi formulado com perguntas concernentes a características socioeconômicas (gênero, idade, estado civil, grau de instrução, renda familiar e profissão) e ainda por perguntas gerais sobre sexualidade, métodos contraceptivos, gravidez, Doenças Sexualmente Transmissíveis, AIDS, além de outras perguntas sociodemográficas (Anexo $1)$.

Os questionários foram respondidos após explanação do pesquisador/aplicador capacitado sobre o objetivo e a natureza da pesquisa, sendo mantidos o anonimato e a confidencialidade dos participantes. Após a aplicação do questionário, os resultados foram devidamente tabulados e analisados.

\section{RESULTADOS E DISCUSSÃO}

A amostra de estudo foi composta por 241 estudantes com predomínio do gênero feminino $(70,9 \%)$. A idade variou de 15 a 29 anos, com predomínio de jovens (15-19 anos). A grande maioria revelou ser solteira. Quando perguntado sobre renda familiar, 46,9\% relatou variar de 2 a 5 salários mínimos. Ainda, quando questionados sobre a profissão, $79,3 \%$ declararam serem estudantes, enquanto o restante $(20,7 \%)$, afirmaram terem outras ocupações além de estudarem. Em relação ao questionamento sobre o conhecimento geral sobre as DSTs pelos adolescentes, 91,3\% responderam e 
demonstraram que conhecem o agente etiológico responsável pelo AIDS, enquanto que $8,7 \%$ afirmaram se tratar de uma bactéria, fungo e/ou protozoário.

A adolescência representa uma fase de vulnerabilidade às DSTs e a AIDS, o que é decorrente de características da própria fase da vida, especialmente a falta de experiência na tomada de decisões e dificuldades no desenvolvimento de relacionamentos a nível social e afetivo (OLIVEIRA et al., 2009). Em um estudo similar de levantamento do conhecimento sobre a AIDS entre alunos adolescentes em fase escolar no leste da Etiópia, Oljiraet al., (2013) constataram que a grande maioria dos jovens entrevistados provinham de famílias com nível socioeconômico mediano a alto, o que os autores correlacionaram com uma certa facilidade ao acesso a informação sobre HIV/AIDS, especialmente pela mídia ou no ambiente escolar. Os resultados encontrados em nosso estudo evidenciam que mesmo indivíduos de nível socioeconômico relativamente intermediário, parcela significativa do total amostral, exibiam de forma geral uma noção aceitável e adequada sobre AIDS e DSTs (91,3\% sabiam qual era o agente causador da doença), evidenciando que o acesso a informação sobre o tema é amplo e de senso comum, relativamente independente da condição social.

Para a análise sobre o conhecimento das vias de transmissão pelos adolescentes, o questionário empregou perguntas indiretas sobre as mesmas, e assim, 94,2\% reconhecem a via sexual como forma de transmissão do HIV, afirmando que o uso de preservativos é eficiente na proteção individual contra o vírus e outras DSTs; 96,7\% afirmam que a via parenteral é uma via importante de transmissão do vírus, enquanto que $92,1 \%$ reconhecem a via vertical como uma das possibilidades de transmissão do vírus. Entretanto, apesar do discernimento da maioria dos adolescentes sobre as vias sexual, parenteral e vertical, $20,3 \%$ afirmam que o uso de talheres, banheiro, um aperto de mão é uma via eficiente de transmissão do vírus. Nader et al., (2009) verificaram que 8,0\% dos adolescentes entrevistados em uma escola em Canoas, Rio Grande do Sul ainda acreditam que o beijo e a proximidade de alguém com AIDS consistem em formas de transmissão do vírus. Esses mitos relacionados à transmissão pelo HIV precisam ser desconstruídos no ambiente escolar e destacados pelas autoridades educacionais (MARTINI; BANDEIRA, 2003) uma vez que, quando essas crenças se disseminam facilmente, elas podem fomentar atitudes de cunho preconceituoso.

Em relação aos métodos contraceptivos, 2,9\% dos indivíduos afirmaram que o uso de anticoncepcional é uma forma eficiente de proteção contra as DSTs. Camargo e Ferreira, encontraram um desconhecimento acima de 
$50 \%$ entre adolescentes quando o assunto era o uso de métodos contraceptivos, o que foi especialmente refletido pela ausência de diferença estatística entre as respostas positivas para o questionamento preservativo e anticoncepcional oral previnem as DSTs. Em sua pesquisa de levantamento sobre o conhecimento geral sobre DSTs com alunos de uma escola do interior Pernambucano, Moreira et al., (2012) comentam que a pílula do dia seguinte foi citada como forma de se prevenir essas doenças, citado por alunas cujas mães fazem uso da mesma. Em um estudo de avaliação do conhecimento contraceptivo de adolescentes grávidas, Duarte et al., (2012) notaram que mesmo o anticoncepcional oral sendo o mais utilizado, entre aquelas que afirmaram empregar algum método contraceptivo, muitas não tinham ciência do mecanismo de ação do mesmo, com uma parcela representativa das entrevistadas afirmando que o anticoncepcional agia matando espermatozóides. Essa noção pode até mesmo alimentar uma ideia errônea que essa forma de contracepção pode auxiliar na eliminação de organismos causadores de DSTs, demonstrando a necessidade de esclarecimentos educativos da função exata desse método.

Apenas $\quad 1,2 \%$ dos indivíduos afirmaram que o uso de preservativo não é uma forma de proteção contra as DSTs. Mesmo diante de um resultado satisfatório como esse, ainda existe uma distância grande na comunidade jovem entre entender a imprescindibilidade do preservativo e o emprego do mesmo. Recentemente, em um trabalho com adolescentes da cidade do interior de Minas Gerais, Mouraet al., (2013), verificaram que o conhecimento sobre HIV/AIDS não foi significativamente associado com um comportamento sexual seguro, demonstrado pelas proporções obtidas de uso de camisinha e número de parceiros sexuais. Em outras palavras, a maior parte da população jovem compreende a importância do uso do preservativo como forma de contracepção e prevenção de DSTs, o que foi observado no presente estudo, contudo essa consciência não se transparece em sua vida sexual, elevando a probabilidade de contrair essas doenças.

Dentre os participantes do estudo, $88,8 \%$ afirmaram que conhecem outros agentes etiológicos causadores de outras DSTs, além do HIV, no entanto $18,7 \%$ não souberam citar algum outro agente. Para os que mencionaram, os mais citados foram: Treponema pallidum (sífilis), Neisseria gonorrhoeae (gonorreia), vírus herpes simplex(HSV), Papilomavírus Humano (HPV), hepatites virais, Haemophilus ducreyi (cancro mole) e Chlamydia trachomatis. Em uma avaliação dos conhecimentos, atitudes e comportamento de adolescentes em uma escola pública do interior gaúcho, Theobaldet al., (2012) listaram que, após a AIDS, as 
DSTs mais mencionadas entre os jovens foram a sífilis e gonorreia, resultado em comum com o atual trabalho, seguida de condiloma, candidíase, cancro mole, tricomoníase e vaginose bacteriana. Brêtas et al.,(2009) comentam que, apesar do percentual significativo de respostas positivas dos jovens estudantes entrevistados com relação ao conhecimento sobre AIDS, gonorreia, sífilis e herpes genital, foi preocupante a falta do mesmo quando se voltou para outras DSTs, como candidíase e tricomoníase, as quais não são exclusivamente transmitidas pela via sexual, e esse desconhecimento também foi verificado sobre as formas de transmissão, sinais e sintomas da herpes genital e condiloma humano, principalmente entre indivíduos do sexo masculino.

O presente estudo evidenciou um nível satisfatório de conhecimento sobre AIDS/DSTs entre os entrevistados, os quais eram constituídos majoritariamente de estudantes adolescentes. Os resultados obtidos exprimem que campanhas de conscientização, fundamentalmente em âmbito escolar, têm surtido efeito e que a população jovem está se tornando cada vez mais informada sobre o controle e prevenção de DSTs. Todavia, algumas observações devem ser feitas, especialmente com relação a falta de entendimento encontrada com relação a vias de transmissão da AIDS e sobre métodos contraceptivos, demonstrando, por outro lado, que o assunto deve ser melhor abordado, com mais detalhamentos em uma linguagem cognoscível para esse público-alvo. Thippeswamy e Gorvine (2012) argumentam que estudos com emprego de questionários para levantamento da compreensão de jovens sobre HIV/AIDS têm revelado que os adolescentes frequentemente possuem informações acuradas sobre vários aspectos do vírus. Entretanto, um aprofundamento nas perguntas mostra informações erradas e confusão sobre aspectos simples da doença. Esses problemas serão solucionados mediante participação ativa e em conjunto das autoridades governamentais e educacionais, as quais devem persistir para que o conhecimento transmitido e adquirido por esses jovens seja verdadeiramente aplicado em suas vidas. Joneset al., (2013) salientam a importância desse tipo de estudo no levantamento de conhecimentos básicos sobre os DSTs e atitudes preventivas relacionadas ao sexo entre adolescentes e jovens, de modo que os próprios resultados obtidos nessas pesquisas possam ser empregados para sustentar e orientar na implementação e melhora das campanhas de de educação e promoção da saúde sexual de jovens em parceria com as entidades governamentais.

\section{CONCLUSÃO}

Em conclusão, os dados aqui apresentados demonstram a falta de conhecimento dos adolescentes sobre as DSTs 
em alguns pontos, e ainda, demonstram o conhecimento básico de grande parte dos participantes da pesquisa sobre métodos de proteção contra DSTs. Ainda, nossos achados sugerem que as campanhas de sensibilização, principalmente no ambiente escolar, têm sido eficazes e destacamos a importância da manutenção e aprimoramento das mesmas, de uma maneira mais lúdica para que todas as dúvidas e mitos possam ser plenamente esclarecidos.

\section{REFERÊNCIAS}

BARBOSA, R. G.; GARCIA, F. C. P.; MANZATO, A. J.; MARTINS, R. A.; VIEIRA, F. T. Conhecimento sobre DST/AIDS, hepatites e conduta sexual de universitários de São José do Rio Preto, SP.Jornal brasileiro de Doenças Sexualmente Transmissíveis, v. 18, n. 4, p.224-230, 2006.

BERGER, D.; BERNARD, S.; KHZAMI, S. E.; SELMAOUI, S.; CARVALHO, G. Sex Education: Teachers' and Future Teachers' Conceptions and Social Representations; What Relevance for Theachers' Training?

XIII. IOSTE Symposium, The Use of Science and Technology Education for Peace and Sustainable Development, p. 2126, 2008.

BRÊTAS, J. R. S.; OHARA C. V. S.; JARDIM, D. P.; MUROYA, R. L. Conhecimento sobre DST / AIDS por estudantes adolescentes. Revista da Escola de Enfermagem da USP, v. 43, n. 3, p. 551557, 2009.

CAMARGO, E. A. I.; FERRARI, R. A. P. Adolescentes: conhecimentos sobre sexualidade antes e após a participação em oficinas de prevenção. Ciência e Saúde

Coletiva, v. 14, n. 3, p. 937-946, 2009.

CARLINI-COTRIM, B.; GAZALCARVALHO, C.; GOUVEIA, N. Health behavior among students of public and private schools on São Paulo, Brazil. Revista de

Saúde Pública, v. 34, n. 6, p. 636-645, 2000.

DUARTE, C. F.; HOLANDA, L. B.; MEDEIROS, M. L. Avaliação de conhecimento contraceptivo entre adolescentes grávidas em uma unidade básica de saúde do Distrito Federal. Journal of the Health Sciences Institute, v. 30, n. 2, p. 140143, 2012.

GERHARDT, C. R.; NADER, S. S.; PEREIRA, D. N. Doenças

SexualmenteTransmissíveis: conhecimento, atitudes e comportamento entre os adolescentes de uma escola pública. Revista Brasileira Médica Famaceutica e Comunitária, v. 3, n. 12, p. 257-270, 2008.

JONES, V.; MODESTE, N.; MARKASH, H. H.; FOX, C. The effect of HIV/AIDS education on adolescents in Trinidad and Tobago. ISRN Infectious Diseases, v. 13, n. 1, p. 1-8, 2013.

KINCAID, C.; JONES, D. J.; STERRETT, E.; McKEE, L. A review of parenting and adolescent sexual behavior: the moderating role of gender. Clinical Psychology Review, v. 32, n. 3, p. 177-188, 2012.

MOREIRA, S. B.; PEREIRA, P. S.; BRITO, A. M.; DUARTE, A. E.; BARROS, L. M. DSTs: percepção de estudantes da escola São Vicente de Paula, Exu-PE. Enciclopédia Biosfera, v. 8, n. 15, p. 2078-2088, 2012.

MOURA, L. R.; LAMOUNIER, J. R.; GUIMARÃES, P. R.; DUARTE, J. M.; BELING, M. T. C.; PINTO, J. A.; GOULART, E. M. A.; GRILLO, C. F. C. A lacuna entre o conhecimento sobre HIV/AIDS e comportamento sexual: uma investigação com adolescentes em Vespasiano, Minas 
Gerais, Brasil. Cadernos de Saúde Pública, v. 29, n. 5, p. 1008-1018, 2013.

OLIVEIRA, D. C.; GOMES, A. M. T.; PONTES, A. P. M.; RIBEIRO, M. C. M.

Conhecimentos e práticas de adolescentes acerca de HIV/AIDS em duas escolas públicas municipais do Rio de Janeiro. Escola Anna Nery Revista de Enfermagem, v. 13, n. 4, p. 833-841, 2009.

OLJIRA, L.; BERHANE, Y.; WORKU, A. Assessment of comprehensive HIV/AIDS knowledge level among in-school adolescents in eastern Ethiopia. Journal of the

International AIDS Society, v. 16, p. 1-5, 2013.

NADER, S. S.; GERHARDT, C. R.; NADER, P. J. H.; PEREIRA, D. N. Juventude e AIDS: conhecimento entre os adolescentes de uma escola pública em Canoas, RS. Revista da Associação Médica do Rio Grande do Sul, v. 53, n. 4, p. 374-381, 2009.

MARTINI, J. G.; BANDEIRA, A. S. Saberes e práticas dos adolescentes na prevenção das Doenças Sexualmente Transmissíveis.

Revista Brasileira de Enfermagem, v. 56, n. 2, p. 160-163, 2003.

ROMERO, K. T.; MEDEIROS, É. H. G. R.; VITALLE, M. S. S.; WEHBA, J. O Conhecimento das adolescentes sobre questões relacionadas ao sexo. Revista da
Associação Médica Brasileira, v. 53, n. 1, p. 51-56, 2007.

SHARP, P. M.; BAILES, E.; ROBERTSON, D. L. GAO, F.; HAHN, B. H. Origins and Evolution of AIDS Viruses. Biological bulletin, v. 196, n. 3, p. 338-342, 1999.

SIRACUSANO, S.; SILVESTRI, T.;

CASOTTO, D. Sexually transmitted diseases: epidemiological and clinical aspects in adults. Urologia, v. 81, n. 4, p. 200-208, 2014.

THEOBALD, V. D.; NADER, S. S.; PEREIRA, D. N.; GERHARDT, C. R.; OLIVEIRA, F. J. M. A universidade inserida na comunidade: conhecimentos, atitudes e comportamentos de adolescentes de uma escola pública frente a doenças sexualmente transmissíveis. Revista da Associação Médica do Rio Grande do Sul, v. 56, n. 1, p. 26-31, 2012.

THIPPESWAMY, G.; GORVINE, B. A qualitative assessment of HIV/AIDS-related knowledge in inner-city minority students. Journal of AIDS and HIV Research, v. 4, n. 3, p. 85-93, 2012.

VIEIRA, L. M.; SAES, S. O.; DÓRIA, A. A. B.; GOLDBERG, T. B. L.Reflexões sobre a anticoncepção na adolescência no Brasil Considerations on contraceptive methods used by adolescents in Brazil. Revista Brasileira de Saúde Materno Infantil, v. 6, n. 1, p. 135-140, 2006. 
Anexo 1 - Questionário aplicado aos adolescentes participantes do "ESPAÇO DAS PROFISSÕES - 2013".

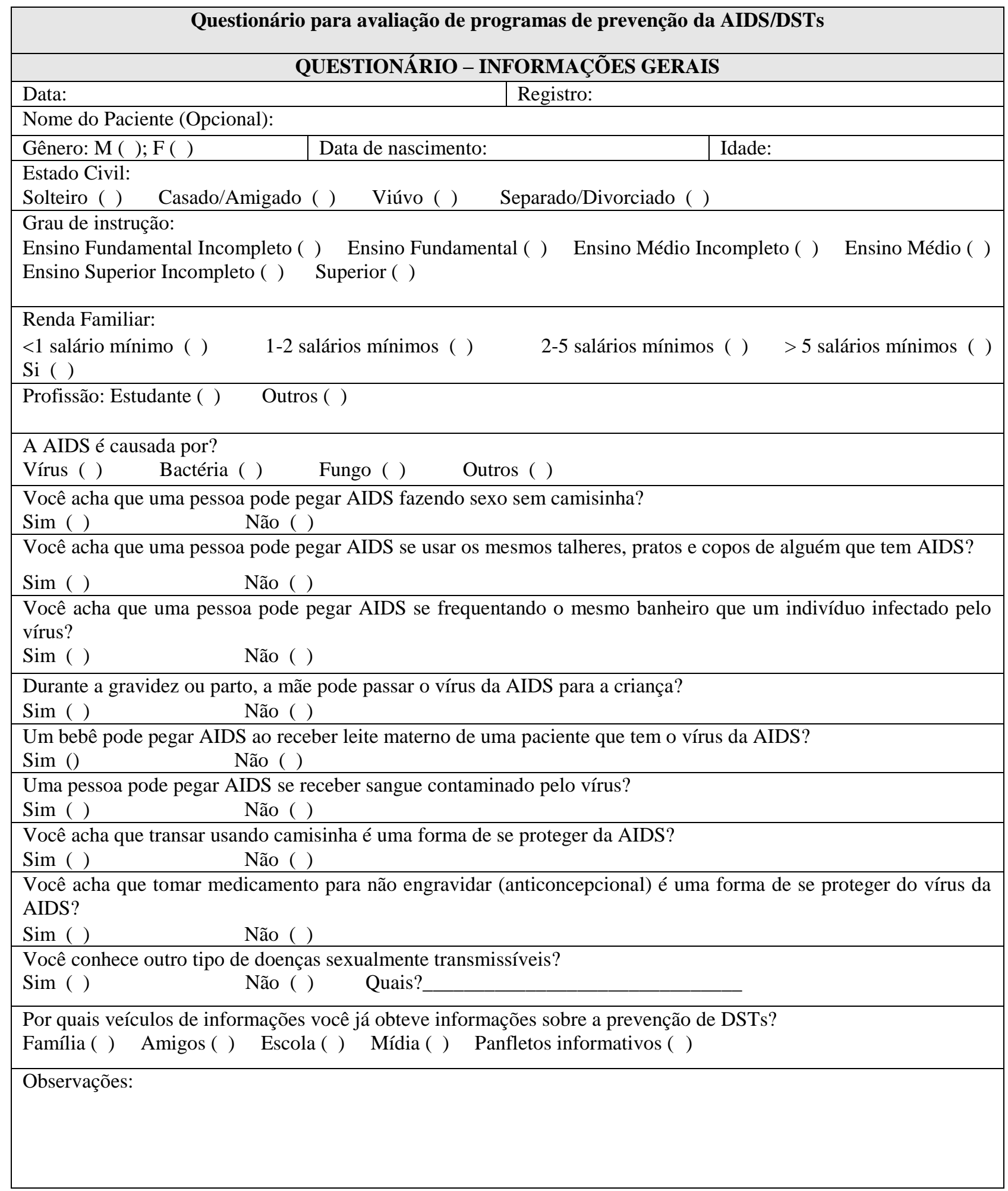

